\title{
Deposition Technology of Metal Matrix Tungsten and Cobalt Alloy
}

\author{
Haipeng Lu
}

\author{
College of Mechanical Engineering, No.97, Heping West Road, Shijiazhuang City Hebei Province, \\ China
}

Keywords: Tungsten cobalt alloy, Electrodeposition technology, Complex surface.

\begin{abstract}
When the metal works at harsh environment, such as high temperature, corrosive gases, ablative particles and mechanical extrusion, abrasion resistance and corrosion resistance of it is needed to extend service life. Though hard chrome coating is used to do this job, there are still many difficult problems, such as serious environmental pollution, physical defects and low energy utilization. However tungsten cobalt alloy has excellent performance in corrosion resistance, high temperature and wear resistance. Because of these characteristics, tungsten cobalt alloy gradually attracts the attention of all walks of life. This paper is mainly for the preparation of tungsten cobalt allot plating formula and the establishment of reliable surface electrodeposition process index system.
\end{abstract}

\section{Introduction}

Tungsten and cobalt alloys were prepared in an electrolytic manner by Holt and his collesgues. Electroplating solution in the electrolyte mainly includes nickel sulfate, cobalt sulfate and sodium tungstate whose effect is complexing agent. Through this method, the alloy has a tungsten content of 35 percent. The tungsten cobalt alloy was then obtained by chemical deposition by Naoe who studied its heat resistance and magnetic properties of the coating film at the same time. B.N. Maruthi electrochemically crystallized to obtain tungsten and cobalt alloys in cobalt sulfate and tungstate electrolytes. And the nanoscale tungsten cobalt alloy is electrodeposited from the acidic solution by Abdel Hamid.

Li Yong and Zhu Yinglu prepare tungsten cobalt alloy by co-deposition method and study the technological conditions of the preparation process and the difficulty of plating the alloy on different matrix materials. Chen Ying carried electroplating to obtain tungsten cobalt alloy and studied the effect of complexing agent, current density and ion concentration on the metal content and properties of the coating. But most people on this technology are also focused on the formulation of the bath.

\section{Tungsten Cobalt Alloy Deposition Principle}

During the alloy co-deposition process, the positive metal is deposited first. As a result, the key to preparing the tungsten cobalt alloy coating is to ensure that the precipitation potential of the tungsten cobalt ions in the electrolyte solution is equal or very close. In order to make the deposition potential consistent, the method that changing the tungsten cobalt ion concentration, adjusting the current density applied at the time of deposition, or adding a complexing agent to the electrolyte is used ${ }^{[1,2]}$.

The higher the cobalt ion concentration, the higher the tungsten content in the tungsten content in the tungsten cobalt alloy. When the potential of the tungsten cobalt electrode is close, what can be done is reducing the tungsten cobalt ion concentration of the positive standard electrode potential or increasing the tungsten ion concentration of the negative standard electrode potential to ensure that tungsten cobalt ions in the cathode electrode material on the reduction of the same potential which can achieve the co-deposition of tungsten cobalt alloy. In the co-deposition, the application of the appropriate current density is to make precipitation potential of tungsten cobalt ion same. When the current density is high, the obtained tungsten cobalt alloy is relatively rough. When the current density is low, although the tungsten cobalt alloy crystal will be more detailed, the cobalt content of cobalt in the alloy is low.

The electrode potential can be affected the complexing agent. The addition of a complexing agent to the electrolyte causes the deposition potential of the metal to become negative. Sodium citrate is 
added to the electrolytic solution containing cobalt sulfate and sodium tungstate as the main components. It can react with cobalt to make the cobalt ion electrode potential negative, which makes cobalt ion electrode potential shift negatively to achieve tungsten cobalt co-deposotion. At the same time, complexing agent can improve the content of cobalt in the alloy. The greater the amount of cobalt added to the electrolyte, the greater the cobalt content.

\section{Main Contents}

The electrochemical process of tungsten and cobalt co-deposition is the theoretical support for the improvement of electroltsis process in the aqueous solution system with sodium sulfate and cobalt sulfate as the electrolyte. The nucleation mechanism of tungsten and cobalt alloy prepared by coprecipitation, co-deposition potential and tungsten-cobalt ion electrochemical reduction mechanism are studied to depth understanding of tungsten cobalt co-deposition process.

For key process factors, the reliability is calculated under different plating process parameters. The mathematical model of process reliability and process parameters is established by using neural network and genetic algorithm and particle swarm optimization algorithm were used to obtain the best electroplating process parameters according to the reliability of different process parameters.

\section{Innovation}

Tungsten cobalt alloy electrodeposition technology is more mature. The bath formulation is suitable for complex surface construction. According to the characteristics of electroplating process, a reasonable and effective characterization process reliability evaluation index system are established. According to the mathematical model of process reliability and process parameters, a mass production process parameter scheme for improving process reliability is proposed.

\section{Difficulty in Tungsten and Cobalt Co-deposition}

Although the realization of the tungsten cobalt alloy preparation, only stay in the process conditions and alloy performance ${ }^{[3]}$. The basic research on the preparation of tungsten and cobalt alloys by co-deposition is not enough. There is no reference value for co-deposition of tungsten ions and cobalt ions. There is no study on the effect of cathodic materials on the deposition potential of tungsten and cobalt ions. And there are few studies on the electrochemical mechanism of cobalt ion ion induced tungsten ion co-deposition ${ }^{[4]}$.

It is difficult to ensure that the deposition thickness is uniform if the surface structure is complex or the space is small. In addition, it is difficult to achieve mass production.

There are many factors that affect the reliability of the process. And the range of parameters is wide. How to select the range of process factors, the level of value, how to select the experimental design method to carry out the test, and then select the key process factors is a major challenge ${ }^{[5-7]}$.

After the key process factors are selected, the relationship between process reliability and process parameters is generally not linear. At the same time there is a certain coupling between the process parameters. So how to clean up the intricate relationship between the two and the establishment of an effective mathematical model are major challenges.

\section{Drugs and Instruments (Table 1)}

Table 1. Drug and instrumentation instructions

\begin{tabular}{|c|c|c|}
\hline Drug Name & Chemical Formula & Specification \\
\hline Sodium Tungstate & $\mathrm{Na}_{2} \mathrm{WO}_{4} \cdot 2 \mathrm{H}_{2} \mathrm{O}$ & A.R. \\
\hline Cobalt Sulfate & $\mathrm{CoSO} \mathrm{O}_{4} \cdot 7 \mathrm{H}_{2} \mathrm{O}$ & A.R. \\
\hline
\end{tabular}




\section{Equipment (Table 2)}

Table 2. Equipment instructions

\begin{tabular}{|c|}
\hline Equipment Name \\
\hline Electrochemical Workstation \\
\hline Electronic Balance \\
\hline Anti-interference AC Purification Power Supply \\
\hline Collector Type Agitator \\
\hline
\end{tabular}

\section{Test Process}

The electrodes and electrolytes are prepared before the test. Insert the motor pole into the electrolyte and fix it. Connect the three electrodes to the electrochemical workstation. The electrochemical behavior of tungsten and cobalt ions alone are investigated. The electrochemical mechanism of tungsten ion co-deposition of tungsten-cobalt alloy is studied (Figure 1).

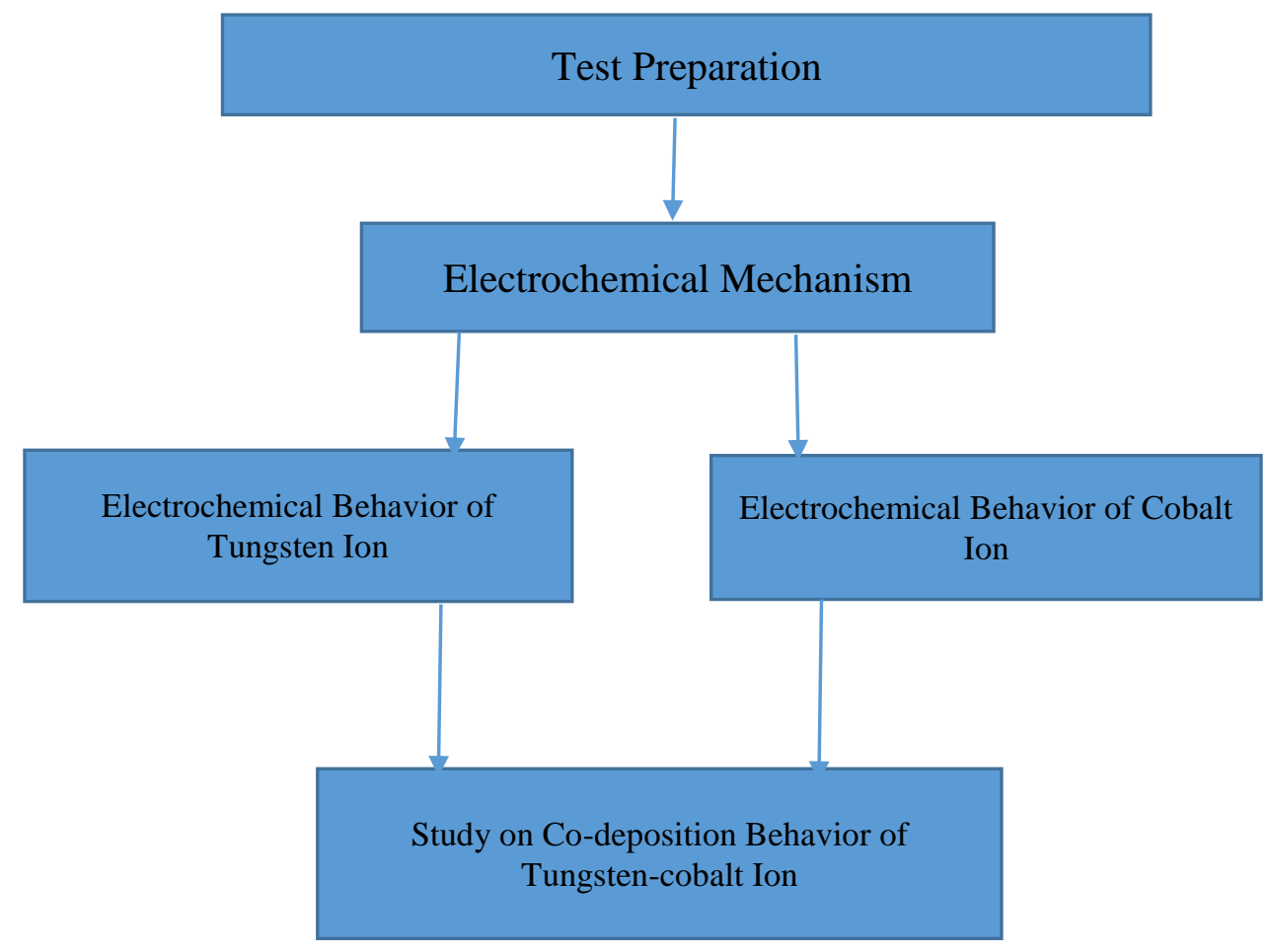

Figure 1. The electrochemical mechanism of tungsten ion co-deposition of tungsten-cobalt alloy

\section{Conclusion}

Though hard chrome coating is used to do this job, there are still many difficult problems, such as serious environmental pollution, physical defects and low energy utilization. However tungsten cobalt alloy has excellent performance in corrosion resistance, high temperature and wear resistance. Because of these characteristics, tungsten cobalt alloy gradually attracts the attention of all walks of life. This paper is mainly for the preparation of tungsten cobalt allot plating formula and the establishment of reliable surface electrodeposition process index system. 


\section{Acknowledgments}

In the course of the study, thanks Ma Wanpeng for advice on research methods. And thanks my classmate who are actively discussing with me when I encounter problems.

\section{References}

[1] HUANG Lihong,GE Hongliang. Study on the Properties of Electrodeposited Tungsten Cobalt Alloy Plating Bath[J]. Surface technology, 2003,32(3):19-20

[2] Mam Ibrahim, S.S Abd, E.L Rehim, S.O Moussa. Electerodeposition of noncrystalline cobalttungsten alloys from citrate electrolytes [J]. Journal of Applied Electrochemistry, 2003 , 33: 627633

[3] LV Chenkai. Electrodeposited Co-based Alloy Coating on Ultrasonic Field and Prediction Model of Related Process Parameters[D]. Suzhou:Suzhou University,2014

[4] XIE Baoru. Electrochemical Behavior of Tungsten Cobalt Co-Deposition[J]. Jiangxi University of Science and Technology,2015

[5] Tanabe T, Wada M. Application of tungsten for plasma limiters in TEXTOR [J]. Journal of Nuclear Materials, 2000,(283-287):1128

[6] Ryu H J, Hong S H. Fabrication and properties of mechanically alloyed oxide-dispersed tungsten heavy alloys [J]. Materials Science\&Engineering A,2003,363:179

[7] Kimura, Kato T, Hyodo T, et al. Electromagnetic Wave Absorption Properties of Carbonyl Irorrferrite PMMA Composites Fabricated by Hybridization Method [J]. Journal of Magnetism and Magnetic Materials ,2007, 312(1): 181-186 